\title{
Canonical Partition Functions of Hamiltonian Systems and the Stationary Phase Formula
}

\author{
J. P. Francoise \\ Bâtiment de Mathématiques, Université de Paris-Sud, F-91405 Orsay Cedex, France
}

\begin{abstract}
We show that there is a Symplectic Action of a Torus associated to Harmonic flows on the Cotangent Bundle of a semi-simple Lie Algebra. This allows to obtain a completely classical proof of the Gallavotti-Marchioro Formula by the method of the Stationary Phase.
\end{abstract}

We compute the Canonical Partition Function,

$$
Z(\beta)=\int_{V^{2 m}} \exp (-\beta H) \Omega \quad(\beta>0)
$$

for a class of integrable Hamiltonian systems. $H$ is the Hamiltonian function, $\Omega=\omega^{m} / m$ ! is the volume form associated to a symplectic form $\omega$ of a symplectic manifold $V^{2 m}$. We compute this integral by a singularity analysis and by an adaptation of the Duistermaat-Heckman theorem $[D-H]$ on the exactness of the stationary phase formula. The proof that Berline-Vergne $[B-V]$ gave of this theorem can be adapted to our situation where $V^{2 m}$ is not compact and where the parameter $\beta$ is positive.

We obtain, for instance, the expression of the Canonical Partition Function for integrable systems which are reductions of the Harmonic flow on the cotangent bundle of a semi-simple Lie algebra. To this purpose, we need to check that these systems are associated to an Hamiltonian action of the torus. We show that the Hamiltonian flows of the eigenvalues of the Lax matrix are all periodic.

Our result extends a formula which was precedingly obtained by Gallavotti and Marchioro $[G-M]$ for the Calogero-Moser system with an external quadratic potential which corresponds to the root system of type $A_{m-1}$. Gallavotti and Marchioro got their result by considering the quantum system and then by taking a limit $(\hbar \rightarrow 0)$.

On the contrary, our proof is purely classical; it illustrates how the DuistermaatHeckman formula can be used also to compute thermodynamical sums.

\section{Adaptation of the Stationary Phase Method}

We consider the proof of [B. V.] of the theorem of the stationary phase of Duistermaat and Heckman. 
Let $V$ be an arbitrary $n$-dimensional manifold and let $\xi$ be a vector field on $V$. Let $\mathscr{Q}=\mathscr{2}^{0}+\cdots+\mathscr{Q}^{n}$ be the De Rham complex on $V$, with the differential $d: \mathscr{Q}^{i} \rightarrow \mathscr{2}^{i+1}$. Let $\delta_{\xi}$ be the operator $d+i(\xi)$. By the Cartan formula, we get $\delta_{\xi}{ }^{2}=L_{\xi}$ (Lie derivative along the vector field $\xi$ ).

We assume furthermore that $n=2 m, V$ is symplectic, that there is an Hamiltonian action of the torus $\pi^{m}$ on $V$. Let $J$ be the moment map, we take as $\xi$ a vector field so that $\xi=J^{*}(X)$ for an element $X$ of the Lie algebra of the torus and that the zeroes of $\xi$ are fixed points for the action of the torus.

Let us put on $V$ an invariant Riemannian metric $\langle$,$\rangle . We define then a$ 1-form $\theta$ on the open set $V_{0}=(x \in V / \xi(x) \neq 0)$ as follows:

$$
\theta_{X}(v)=\langle\xi(x), v\rangle /\langle\xi(x), \xi(x)\rangle .
$$

This 1-form has the properties:

i) $L_{\xi} \theta=0$,

ii) $\theta(\xi)=1$.

Lemma 1-1. (Berline-Vergne). Let $\mu=\mu_{2 m}+\cdots \mu_{0}$ be an even element of $\mathscr{G}$ such that $\delta_{\xi} \mu=0$. Then $\mu_{2 m}$ is d-exact on $V_{0}$.

Proof. We introduce

$$
v=\theta \wedge(1+d \theta)^{-1} \wedge \mu .
$$

We get from i) and ii) $i(\xi) d v=i(\xi) \mu$, and since on $V_{0}, i(\xi): \mathscr{Q}^{2 m} \rightarrow \mathscr{Q}^{2 m-1}$ is injective, $\mu_{2 m}=d v_{2 m-1}$.

If we set $\alpha=H-\omega$, where $H$ is the Hamiltonian of $\xi$, then, we have, $\delta_{\xi} \alpha=0$. We apply the lemma to

$$
\mu=\exp -\beta \alpha=\exp (-\beta H)\left(1+B \omega+\cdots+\left(\beta^{m} / m !\right) \omega^{m}\right) .
$$

We get:

$$
\exp (-\beta H) \Omega=d\left(\beta^{-m} v_{2 m-1}\right) .
$$

We assume furthermore that:

- $\left(c_{1}\right) H$ is a rational (respectively meromorphic) positive function on $\mathbb{R}^{2 m}$; it blows up along finitely many algebraic (respectively analytic) subsets of codimension 1 denoted $\Delta_{i}(i=1, \ldots, m)$.

$-\left(c_{2}\right) V=T^{*} W, W=\mathbb{R}^{m} / U_{i=1}^{m} \Delta_{i}, \omega=\Sigma d x_{1} \wedge d y_{i}$

$-\left(c_{3}\right)$ The only critical points of $H$ are minima. This implies that the critical points of $H$ must be fixed points for the action of the torus $\mathbb{T}^{m}$. (otherwise they could not be isolated)

$$
-\left(c_{4}\right) H(x, y) \rightarrow+\infty \text { when }(x, y) \rightarrow \infty \text {. }
$$

Remark. In our examples, $H$ is convex, so that the condition $\left(c_{3}\right)$ is naturally fulfilled.

Let $d(p, \Delta)$ be the distance in $\mathbb{R}^{2 m}$ from a point $p$ to a closed set $\Delta$. We introduce the set:

$$
D_{R}=\left\{(x, y) /\|(x, y)\|<R, d\left((x, y), \Delta_{i}\right)>R^{-1} \quad \text { for all }(i=1, \ldots, s .)\right.
$$


We denote by $C$, the set of the Critical points of the function $H$ and let $B_{p}, p \in C$, be small balls centered about the critical points of $H$. From our assumptions, the Canonical Partition Function of $H$ :

$$
Z(\beta)=\int_{\mathbb{R}^{2 m}} \exp (-\beta H) \Omega
$$

exists and is equal to

$$
Z(\beta)=\lim _{R \rightarrow \infty} \int_{D_{R}} \exp (-\beta H) \Omega .
$$

By Lemma $1-1$, there is a $2 m-1$ form $v_{2 m-1}$ defined by (2) so that by Stokes' theorem, we have,

$$
Z(\beta)=\sum_{p \in C} \int_{B_{p}} \exp (-\beta H) \Omega-\int_{\delta B_{p}} \beta^{-m} v_{2 m-1}+\lim _{R \rightarrow \infty} \int_{\delta D_{R}} \beta^{-m} v_{2 m-1} .
$$

Our situation differs from the Duistermaat-Heckman case as $\beta$ is a positive parameter (versus a purely imaginary one) and as the manifold $V$ is not compact. So we have to show that the limit in (5) is equal to zero. This can be done with the Lojasiewicz inequality $[L]$.

Lemma 1-2. The contribution of the boundary vanishes:

$$
\lim _{R \rightarrow \infty} \int_{\delta D_{R}} \beta^{-m} v_{2 m-1}=0
$$

Proof. $H$ is a rational (respectively meromorphic) function on $\mathbb{R}^{2 m}$, since it blows up either when $(x, y)$ tends to infinity or along the algebraic (respectively analytic) subsets $\Delta_{i}(i=1, \ldots, s)$, the Lojasiewicz inequality $[L]$ shows the existence of a positive constant 1 such that

$$
\exp (-\beta H(x, y))\left\langle\exp \left(-R^{1}\right) \quad \text { for } \quad(x, y) \in \delta D_{R}, R>0 .\right.
$$

From (1), the 1 -form $\theta$ blows up eventually when $(x, y)$ approaches the subsets $\Delta_{i}$; but, again by the Lojasiewicz inequality, it has at most a polynomial growth there.

So that by (2) the form $v_{2 m-1}$ satisfies

$$
\left\|v_{2 m-1}\right\|<c R^{\tau} \exp -R^{1} \text { for some constants } c, \tau>0 \text {. }
$$

Also, we see easily that the volume of $\delta D_{R}$ increases as an algebraic function of $R$ as $R$ tends to infinity. This ends the proof.

We finish now as in $[\mathrm{B}-\mathrm{V}]$. By the canonical form theorem $[\mathrm{G}-\mathrm{S}]$ (from the assumption that the critical points of $H$ are of Morse type and that $H$ is associated to the action of a torus), we can find symplectic coordinates $\left(x_{1}, \ldots, x_{m} ; y_{1}, \ldots, y_{m}\right)$, given by a local diffeomorphism $\Phi$, on the ball $B_{p}$ such that:

$$
\Phi^{*} H=H(p)+\sum_{k=1}^{m} \lambda_{k}(p)\left(x_{k}^{2}+y_{k}^{2}\right)=H_{0} .
$$

If we compute the terms on the right-hand side of (5) by making a second application of Stokes' theorem, we get:

where $v_{2 m-1}{ }^{0}=\Phi^{*}\left(v_{2 m-1}\right)$.

$$
\int_{\Phi^{-1}\left(\boldsymbol{B}_{p}\right)} \exp -\beta H_{0} \Omega-\int_{\delta \Phi^{-1}\left(\boldsymbol{B}_{p}\right)} v_{2 m-1}{ }^{0}=\int_{\mathbb{R}^{2 m}} \exp -\beta H_{0} \Omega,
$$


The right-hand side of Eq. (5) can now be easily computed. We see that $Z(\beta)$ is determined by the critical values of $H$ and by the eigenvalues of the Hessian of $H$ at its critical points. We have now proved the

Theorem 1-3. Under the conditions (c), and with the notations of (7), the Canonical Partition Function of $H$ is equal to:

$$
Z(\beta)=\sum_{p \in C} \pi^{m} \beta^{-m}\left(\prod_{k=1}^{m} \lambda_{k}(p)\right)^{-1} \exp -\beta H(p) .
$$

\section{Hamiltonian Torus Action}

Let $\mathscr{G}$ be a semi-simple Lie algebra of dimension $m$. The cotangent bundle $T^{*} \mathscr{G}$ may be identified with $\mathscr{G} \times \mathscr{G}^{*} \simeq \mathscr{G} \times \mathscr{G}$. As a cotangent bundle, it has a symplectic structure which can be written [K. K. S.]:

$$
\omega=\operatorname{Tr} d X \wedge d L, \quad(X, L) \in \mathscr{G} \times \mathscr{G} .
$$

We define on $T^{*} \mathscr{G}$ the Harmonic Flow by the Hamiltonian Function

$$
H=(1 / 2) \operatorname{Tr}\left(\lambda^{2} X^{2}+L^{2}\right) \text {. }
$$

The group $G$ acts on $\mathscr{G}$ by the adjoint action; this action lifts into an Hamiltonian action on $T^{*} \mathscr{G}$. The Harmonic flow has $m$ commuting independent integrals

$$
H_{k}=\operatorname{Tr}\left(\lambda^{2} X^{2}+L^{2}\right)^{k}
$$

which are $G$-invariant functions.

Following [O. P], a projection of the Hamiltonian system (9) on the cotangent bundle of the Weyl chamber $T^{*} W$, defines the system

$$
\begin{aligned}
& H=(1 / 2) \sum_{i=1}^{m}\left(y_{i}{ }^{2}+\lambda^{2} x_{i}{ }^{2}\right)+(1 / 2) \sum_{\alpha \in A^{+}} g_{\alpha}{ }^{2}|\alpha|^{2} /\langle x, \alpha\rangle^{2}, \\
& \omega=\sum_{i=1}^{m} d x_{i} \wedge d y_{i} .
\end{aligned}
$$

In (10) $A+$ means the set of the positive roots of the Lie algebra $\mathscr{G}$ and the $g_{\alpha}$ are arbitrary positive constants which are equal for two elements $\alpha, \alpha^{\prime}$ which are conjugated under the Weyl group.

\section{Examples:}

1 -If $\mathscr{G}$ is of $A_{m-1}$ type, the walls of the Weyl chamber are given by the hyperplanes

$$
\left(x_{i}=x_{j}, i \neq j\right)
$$

the Weyl group is the permutation group $G_{m}$, it acts transitively on the set $A+$, so all the constants $g_{\alpha}$ must be equal. In that case, we get

$$
H=(1 / 2) \sum_{i=1}^{m}\left(y_{i}^{2}+\lambda^{2} x_{i}^{2}\right)+g^{2} \sum_{i<j}\left(x_{i}-x_{j}\right)^{-2},
$$

which is the Calogero-Moser system with an external quadratic potential. 
2-In the $B_{m}=C_{m}$ case, the walls of the Weyl chambers are given by the hyperplanes

$$
\left(x_{i}=x_{j}, i \neq j\right), \quad\left(x_{i}=-x_{j}, i \neq j\right), \quad\left(x_{i}=0\right) .
$$

The set $A+$ has two orbits under the action of the Weyl group. So that there are two constants $g_{1}$ and $g_{2}$ and that we get

$$
\begin{aligned}
H= & (1 / 2) \sum_{i=1}^{m}\left(y_{i}{ }^{2}+\lambda^{2} x_{i}^{2}\right) \\
& +g_{1}{ }^{2}\left(\sum_{i<j}\left(x_{i}-x_{j}\right)^{-2}+\sum_{i<j}\left(x_{i}+x_{j}\right)^{-2}\right)+g_{2}{ }^{2} \sum_{i=1}^{m} x_{i}{ }^{-2} .
\end{aligned}
$$

3 - In the $D_{m}$ case, we obtain the Hamiltonian system which corresponds to (12) with $g_{2}=0$.

We explain now in details why we can associate an Hamiltonian torus action to the Calogero-Moser system with an external quadratic potential. We can proceed in quite a similar way for the more general system (10) (type $V$ in the Olshanetsky-Perelomov classification [O. P]) with slightly different notations.

We use the matrices $(X, L)$ defined by:

$$
\begin{aligned}
& X_{i j}=x_{i} \delta_{i j}, \\
& L_{i j}=y_{i} \delta_{i j}+\sqrt{ }-1 g /\left(x_{i}-x_{j}\right)\left(1-\delta_{i j}\right) .
\end{aligned}
$$

We note that we have the relation $[X, L]=\sqrt{ }-1 g C$, where the matrix $C$ is defined by $C_{i j}=1-\delta_{i j}$. Under the Hamiltonian flow of the function $H=(1 / 2)$ $\operatorname{Tr}\left(\lambda^{2} X^{2}+L^{2}\right)$, the matrices $X, L$ have the following evolution:

$$
\dot{X}=[A, X]+L, \dot{L}=[A, L]-\lambda^{2} X,
$$

where $A$ is given by:

$$
A_{i j}=\sqrt{-1} \delta_{i j} \sum_{k \neq i} g /\left(x_{i}-x_{k}\right)^{2}+\left(1-\delta_{i j}\right)(g \sqrt{-1}) /\left(x_{i}-x_{j}\right)^{2} .
$$

We introduce the matrices $Z=\sqrt{ }-1 \lambda X+L$ and $Z^{*}=-\sqrt{ }-1 \lambda X+L$; the matrix $P=Z Z^{*}$ undergoes an isospectral deformation

$$
\dot{P}=[A, P],
$$

and we remark that $Z^{*}={ }^{t} \underline{Z}$ (transposed of the complex conjugated) and accordingly that $P^{*}=P$. The eigenvalues of $P$ are algebraic functions on $T^{*} W$. Let $\Lambda\left(=\Lambda_{p}\right)$ be one of these eigenvalues.

Theorem. The Hamiltonian flow defined by $\Lambda$ on $T^{*} W$ is periodic. All the periodic orbits have the same period (not necessarily primitive) $2 \pi / \lambda$.

Let us introduce the eigenvector $\Psi$ of $P$ for the eigenvalue $\Lambda$. The Hamiltonian flow of $\Lambda$ is defined by the equations:

$$
\begin{gathered}
\left\{\Lambda, x_{i}\right\}=\partial \Lambda / \partial y_{i} \\
\left\{\Lambda, y_{i}\right\}=-\partial \Lambda / \partial x_{i} .
\end{gathered}
$$


The matrix $P$ can be diagonalized by an element $V$ of the unitary group. If we denote by $\langle$,$\rangle the Hermitian product, we get$

$$
\begin{aligned}
& \partial \Lambda / \partial x_{i}=\partial\langle P \Psi, \Psi\rangle / \partial x_{i}=\left\langle\partial P / \partial x_{i} \Psi, \Psi\right\rangle, \\
& \partial \Lambda / \partial y_{i}=\partial\langle P \Psi, \Psi\rangle / \partial y_{i}=\left\langle\partial P / \partial y_{i} \Psi, \Psi\right\rangle .
\end{aligned}
$$

Let us introduce the matrix $T$ :

$$
T_{i j}=\Psi_{i} \Psi_{j}^{*}
$$

(* for the complex conjugation).

Lemma 2-1. The matrix $V^{-1} T V$ is diagonal such that:

$$
V^{-1} T V_{i j}=\Delta_{i j}=\delta_{i p} \delta_{i j} .
$$

Proof. We remind the reader that the index $p$ refers to the eigenvalue $\Lambda$. We have

because

$$
T_{i j}=V \Delta V_{i j}^{-1}=\sum_{1, s} V_{i 1} \Delta_{1 s} V_{s j}^{-1}
$$

$$
T_{i j}=\Psi_{i} \Psi_{j}^{*}=V_{i p} V_{j p}
$$

As a corollary, we get: $[P, T]=0$. We prove now the

Proposition 2-1. Under the Hamiltonian flow of $\Lambda$, the time evolution of $X$ and $L$ is given by the equations:

$$
\begin{aligned}
\dot{X} & =\left[A^{(p)}, X\right]+\{\{L, T\}\}, \\
\dot{L} & =\left[A^{(p)}, L\right]-\lambda^{2}\{\{X, T\}\},
\end{aligned}
$$

where the elements of the matrix $A^{(p)}$ are:

$$
\begin{aligned}
& \quad(i \neq j) A_{i j}=\left(y_{i}+y_{j}\right) /\left(x_{i}-x_{j}\right) T_{i j}+\sqrt{ }-1 g\left(T_{i i}+T_{j j}\right) /\left(x_{i}-x_{j}\right)^{2} \\
& \quad+\sum_{k \neq i, j} \sqrt{ }-1 g T_{k j} /\left(x_{i}-x_{j}\right)\left(x_{i}-x_{k}\right)+\sum_{k \neq i, j} \sqrt{ }-1 g T_{i k} /\left(x_{i}-x_{j}\right)\left(x_{k}-x_{j}\right),
\end{aligned}
$$

and where the symbol $\{\{\}$,$\} means the anticommutator of two matrices.$

Proof. We write the elements of the matrix $P$ :

$$
\begin{aligned}
P_{i i}=\lambda^{2} x_{i}^{2} & +y_{i}^{2}+\sum_{k \neq i} g^{2} /\left(x_{i}-x_{k}\right)^{2}, \\
(i \neq j) P_{i j}= & \sum_{k \neq i, j}-g^{2} /\left(x_{i}-x_{k}\right)\left(x_{k}-x_{j}\right) \\
& +\sqrt{ }-1 g\left(y_{i}+y_{j}\right) /\left(x_{i}-x_{j}\right) .-\lambda g .
\end{aligned}
$$

To check (20), we have first to show that:

$$
\dot{x}_{i}=\{\{L, T\}\}_{i i}=\sum_{j} L_{i j} T_{j i}+L_{j i} T_{i j} .
$$

The sum of the right-hand side is: 


$$
2 y_{i} T_{i i}+\sum_{k \neq i} \sqrt{ }-1 g\left(T_{k i}-T_{i k}\right) /\left(x_{i}-x_{k}\right),
$$

which is $\dot{x}_{i}$ by (18) and (24).

Then for $i \neq j$, we have to prove that

We get:

$$
0=\left[A^{(p)}, X\right]_{i j}+\{\{L, T\}\}_{i j} .
$$

$$
A_{i j}\left(x_{i}-x_{j}\right)=\{\{L, T\}\}_{i j},
$$

which is precisely (22).

Let us see now the diagonal term of (21), we have to check that

$$
\dot{y}_{i}=-\sum_{j \neq i} \sqrt{ }-1 g\left(A_{i j}+A_{j i}\right) /\left(x_{i}-x_{j}\right)-2 \lambda^{2} x_{i} T_{i i},
$$

which is precisely given by (19), (22) and (24).

The off-diagonal terms of (21) deserve the most part of our attention; we get:

$$
\begin{aligned}
& -\sqrt{ }-1 g\left(\dot{x}_{i}-\dot{x}_{j}\right) /\left(x_{i}-x_{j}\right)^{2} \\
& =\sqrt{ }-1 g\left(A_{i i}-A_{j j}\right) /\left(x_{i}-x_{j}\right)+A_{i j}\left(y_{j}-y_{i}\right)+\sum_{k \neq i, j} \sqrt{ }-1 g A_{i k} /\left(x_{k}-x_{j}\right) \\
& \quad-\sqrt{ }-1 g A_{k j} /\left(x_{i}-x_{k}\right)-\lambda^{2}\left(x_{i}+x_{j}\right) T_{i j} .
\end{aligned}
$$

If we multiply by $\left(x_{i}-x_{j}\right) / \sqrt{ }-1 g$, this gives:

$$
\begin{gathered}
-\sum_{k \neq i, j} \sqrt{ }-1 g\left(T_{i k}-T_{k i}\right) /\left(x_{i}-x_{k}\right)\left(x_{i}-x_{j}\right) \\
+\sum_{k \neq i, j} \sqrt{ }-1 g\left(T_{j k}-T_{k j}\right) /\left(x_{j}-x_{k}\right)\left(x_{i}-x_{j}\right)+\cdots \\
\left(y_{i}+y_{j}\right)\left(T_{i i}-T_{j j}\right) /\left(x_{i}-x_{j}\right)+\cdots \\
\sqrt{ }-1 g^{-1}\left(\left(\lambda^{2} x_{i}^{2}+y_{i}^{2}\right)-\left(\lambda^{2} x_{j}^{2}+y_{j}^{2}\right)\right) T_{i j}+\cdots \\
\sum_{k \neq i, j} T_{k j}\left(y_{j}-y_{i}\right) /\left(x_{i}-x_{k}\right)+\sum_{k \neq i, j} T_{i k}\left(y_{j}-y_{i}\right) /\left(x_{k}-x_{j}\right) \\
\sum_{s \neq i, j}\left(A_{i s}\left(x_{i}-x_{j}\right) /\left(x_{s}-x_{j}\right)-A_{s j}\left(x_{i}-x_{j}\right) /\left(x_{i}-x_{s}\right)\right)+\cdots \\
A_{i i}-A_{j j}=0 .
\end{gathered}
$$

To show this equality, we begin to remark that:

$$
\begin{gathered}
\sum_{s \neq i, j}\left(A_{i s}\left(x_{i}-x_{s}\right) /\left(x_{s}-x_{j}\right)-A_{s j}\left(x_{s}-x_{j}\right) /\left(x_{i}-x_{s}\right)\right) \\
\sum_{s \neq i} A_{i s}-\sum_{s \neq j} A_{s j}, \\
\sum_{s \neq i, j} T_{i s}\left(y_{j}+y_{s}\right) /\left(x_{s}-x_{j}\right)-\sum_{s \neq i, j} T_{s j}\left(y_{i}+y_{s}\right) /\left(x_{i}-x_{s}\right) \\
\sum_{s \neq i, j} T_{i s}\left(y_{i}+y_{s}\right) /\left(x_{s}-x_{j}\right)+\sum_{s \neq i, j} T_{s j}\left(y_{s}+y_{j}\right) /\left(x_{i}-x_{s}\right) \\
\sum_{s \neq i, j} T_{i s}\left(y_{i}+y_{s}\right) /\left(x_{s}-x_{j}\right)-\sum_{s \neq i, j} T_{s j}\left(y_{s}+y_{j}\right) /\left(x_{i}-x_{s}\right)+\cdots \\
\sum_{s \neq i, j} \sqrt{ }-1 g\left(T_{i i}-T_{j j}\right) /\left(x_{i}-x_{s}\right)\left(x_{s}-x_{j}\right)+\cdots
\end{gathered}
$$




$$
\begin{gathered}
\sum_{s \neq i, j ; k \neq i, j, s} \sqrt{ }-1 g T_{i k} /\left(x_{k}-x_{s}\right)\left(x_{s}-x_{j}\right)-\sum_{s \neq i, j ; k \neq i, j, s} \sqrt{ }-1 g T_{k j} /\left(x_{s}-x_{k}\right)\left(x_{i}-x_{s}\right) \\
+\cdots \sum_{s \neq i} \sqrt{ }-1 g T_{i j} /\left(x_{i}-x_{s}\right)^{2}-\sum_{s \neq j} \sqrt{ }-1 g T_{i j} /\left(x_{j}-x_{s}\right)^{2}+\cdots \\
\sum_{s \neq i, j} \sqrt{ }-1 g T_{j s} /\left(x_{s}-x_{j}\right)\left(x_{i}-x_{j}\right)-\sqrt{ }-1 g T_{s i} /\left(x_{i}-x_{s}\right)\left(x_{i}-x_{j}\right)
\end{gathered}
$$

We have that:

$$
\begin{aligned}
(29)+(36)= & (34) \\
(27)+(37)= & -\sqrt{ }-1 g^{-1} P_{i j}\left(T_{i i}-T_{j j}\right)-\lambda \sqrt{ }-1\left(T_{i i}-T_{j j}\right), \\
(28)+(39)= & -\sqrt{ }-1 g^{-1}\left(-P_{i i}+P_{j j}\right) T_{i j}, \\
(26)+(40)= & -\sum_{k \neq i, j} \sqrt{ }-1 g T_{i k} /\left(x_{i}-x_{j}\right)\left(x_{i}-x_{k}\right) \\
& -\sum_{k \neq i, j} \sqrt{ }-1 g T_{k j j} /\left(x_{i}-x_{j}\right)\left(x_{j}-x_{k}\right), \\
(38)+(41)= & -\sum_{s \neq i, k ; k \neq l, j} \sqrt{ }-1 g T_{k j} /\left(x_{s}-x_{k}\right)\left(x_{i}-x_{s}\right) \\
& +\sum_{s \neq j, k ; k \neq i, j} \sqrt{ }-1 g T_{i k} /\left(x_{k}-x_{s}\right)\left(x_{s}-x_{j}\right), \\
(34)+(42)= & -\sqrt{ }-1 g^{-1} \sum_{s \neq i, j}\left(P_{s j} T_{i s}-P_{i s} T_{s j}\right) \\
& -\lambda \sqrt{ }-1 \sum_{s \neq i} T_{i s}+\lambda \sqrt{ }-1 \sum_{s \neq j} T_{s j},
\end{aligned}
$$

and so finally:

$$
\begin{aligned}
(27)+ & (28)+(31)+(32)+(30)+(34)+(37)+(39)+(42) \\
= & \sqrt{ }-1 g^{-1}[P, T]_{i j}+\sum_{s \neq i} A_{i s}-\lambda \sqrt{ }-1 \sum_{s \neq i} T_{i s}-\sum_{s \neq j} A_{s j} \\
& +\lambda \sqrt{ }-1 \sum_{s \neq j} T_{s j}-\lambda \sqrt{ }-1 T_{i i}+\lambda \sqrt{ }-1 T_{j j}+A_{i i}-A_{j j}=0 .
\end{aligned}
$$

Note that to end the proof of the proposition, we have to check the coherence of (23), that is:

$$
\sum_{s \neq i} A_{i s}-\lambda \sqrt{ }-1 T_{i s}=\sum_{s \neq i} A_{s i}-\lambda \sqrt{ }-1 T_{s i},
$$

but this is a direct consequence of $\sqrt{ }-1 g^{-1}[P, T]_{i i}=0$.

From (20) and (21), and again the fact that $[P, T]=0$, we deduce that the matrix $P$ is isospectral for the flows of its eigenvalues. We find by this way that the eigenvalues of $P$ are in involution.

At this point, we introduce the matrix $U$, solution of the differential equation:

$$
\dot{U}=A U
$$

so that $U(\infty)=1$ (identity matrix).

For imaginary values of $\lambda$, we have unbounded orbits for the flow of the Hamiltonian, and so $Z, Z^{*}$ and $P=Z Z^{*}$ tend to diagonal matrices. We have,

$$
d U P U^{-1} / d t=0 \text { and so, }
$$


$U P U^{-1}=U(\infty) U^{-1}(\infty)$ is a diagonal matrix also for real values of $\lambda$. Since $T$ commutes with $P, U T U^{-1}$ is diagonal, and by Lemma $2-1$, we have necessarily, $U T U^{-1}=\Delta$.

We introduce now the matrices:

$$
\xi=U Z U^{-1}, \quad \xi^{*}=U Z^{*} U^{-1} .
$$

to write that (20) and (21) imply

$$
\dot{\xi}=\sqrt{ }-1 \lambda\{\{\xi, \Delta\}\}, \quad \dot{\xi}^{*}=-\sqrt{ }-1 \lambda\left\{\left\{\xi^{*}, \Delta\right\}\right\} .
$$

These equations can be integrated into:

$$
\dot{\xi}(t)=e^{\sqrt{ }-1 \lambda t \Delta} \xi(0) e^{\sqrt{ }-1 \lambda t \Delta}, \quad \xi^{*}(t)=e^{-\sqrt{ }-1 \lambda t \Delta \xi^{*}}(0) e^{-\sqrt{ }-1 \lambda t \Delta} .
$$

By taking the difference, we get that $X(t)=U X U^{-1}$ is a periodic matrix of period $2 \pi / \lambda$. The traces of $X(t)^{j}$ for all integers $j$ are periodic functions of $t$ of period $2 \pi / \lambda$. We obtain that all the functions $x_{i}(t)$ are periodic of period $2 \pi / \lambda$ (of course, it is not necessarily the primitive period.)

We conclude now this paragraph. The Hamiltonian flows generated by the functions $\Lambda_{1}, \ldots, \Lambda_{m}$ commute to each other and are periodic.

They define an Hamiltonian Action of the torus $\mathbb{T}^{m}$ on $T^{*} W$, the moment map $J$ being given by

$$
J: T^{*} W \rightarrow \mathbb{R}^{m}(x, y) \rightarrow\left(t_{1}, \ldots, t_{m}\right)=\left(\Lambda_{1}(x, y), \ldots, \Lambda_{m}(x, y)\right) .
$$

The Hamiltonian system which defines the Calogero-Moser system with an external quadratic potential, or more generally the Olshanetsky-Perelomov systems of type $V$, is given by

$$
H=\Lambda_{1}+\cdots+\Lambda_{m},
$$

so that for the element $X=(1, \ldots, 1)$ of $\left(\mathbb{T}^{m}\right)^{*}$, the Hamiltonian vector field $\xi$ of $H$ satisfies $\xi=J^{*}(X)$. We can apply the method of the paragraph (1) to compute its Canonical Partition Function.

\section{Generalizations of the Gallavotti-Marchioro Formula}

From $[\mathrm{C}]$ and $[\mathrm{P}]$, we can deduce the following analysis of the set of critical points of $H$ given by (10). Since $H$ is convex, it has only one critical point in each connected component of the Weyl chamber and it is a minimum; the value of this minimum is equal to $H=\lambda \sum_{\alpha \in R_{+}} g_{\alpha}$. At each of these minima, the Hessian of $H$ is equal to:

$$
\text { (1/2) } \sum_{i=1}^{m}\left(y_{i}^{2}+\lambda^{2} v_{i}{ }^{2} x_{i}^{2}\right) \text {, }
$$

where $v_{i}(i=1, \ldots, m)$ denote the degrees of the basic invariants of the Weyl group $[\mathrm{Ch}]$. We can now prove the

Theorem 3-1. Let $H$ be the Hamiltonian system given by (10), the Canonical Partition Function $Z(\beta)$ of $H$ is given by: 


$$
Z(\beta)=(2 \pi / \beta \lambda)^{m} \exp -\left(\beta \lambda \sum_{\alpha \in A^{+}} g_{\alpha}\right) .
$$

Proof. There is a symplectic system of coordinates $\left(p_{i}, q_{i}\right)$ so that

$$
y_{i}=\left(\lambda v_{i}\right)^{1 / 2} p_{i}, \quad q_{i}=\left(\lambda v_{i}\right)^{1 / 2} x_{i}, \quad H=\lambda \sum_{\alpha \in A+} g_{\alpha}+(\lambda / 2) \sum_{i=1}^{m} v_{i}\left(p_{i}^{2}+q_{i}^{2}\right) .
$$

The formula (8) gives for each connected component of the Weyl chamber, a contribution

$$
(2 \pi / \beta \lambda)^{m}\left(v_{1} \cdots v_{m}\right)^{-1} \exp -\left(\beta \lambda \sum_{\alpha \in R_{+}} g_{\alpha}\right)
$$

to the integral $Z(\beta)$. The order of the Weyl group is equal to the product $v_{1} \cdots v_{m}$ $[\mathrm{Ch}]$, and so we obtain the announced formula.

\section{Examples:}

1 - In the $A_{m-1}$ case, we obtain

$$
Z(\beta)=(2 \pi / \beta \lambda)^{m} \exp -(\beta \lambda g m(m-1) / 2)
$$

which is the Gallavotti-Marchioro formula [G. M.].

2 - For the $B_{m}=C_{m}$ case, we have:

$$
Z(\beta)=(2 \pi / \beta \lambda)^{m} \exp -\beta\left(g_{1} \lambda m(m-1)+g_{2} \lambda m\right) .
$$

3 - The case $D_{m}$ corresponds to $g_{2}=0$.

Acknowledgement. The author thanks the referee for providing him with the reference [G.], where another classical proof of the Gallavotti-Marchioro formula in the case of two particles and for the Lie Algebra $A_{m-1}$ was given.

\section{References}

[A] Adler M.: Some finite dimensional integrable systems and their scattering behavior. Commun. Math. Phys. 55, 195-230 (1977)

[B. V.] Berline N., Vergne M.: Zeros d'un champ de vecteurs et classes caractéristiques équivariantes, Duke Math. J. 50, 539-549 [1983]

[C] Calogero F.: Equilibrium configuration of the one-dimensional $n$-Body problem with Quadratic and Inversely Quadratic Pair Potentials, Lett. Nuovo Cimt. 20, 251-253 (1977)

[Ch] Chevalley C.: Théorie des groupes de Lie. Paris: Hermann 1968

[D. H.] Duistermaat J. J., Heckman G. H.: On the variation in the cohomology of the symplectic form of the reduced phase space. Invent. Math. 69, 259-268 (1982)

[G. M.] Gallavotti G., Marchioro C.: On the calculation of an integral. J. Math. Anal. Appl. 44, 661675 (1973)

[G] Glasser M. L.: A remarkable property of definite integrals. Math. Comput. V. 40, No. 162, 561-563 (1983)

[G. S.] Guillemin V., Sternberg S.: Symplectic techniques in physics. Cambridge: Cambridge University Press 1984

[K. K. S.] Kazhdan J., Kostant B., Sternberg S.: Hamiltonian group actions and dynamical systems of Calogero type. Commun. Pure Appl. Math. 31, 481-508 (1978)

[L] Lojasiewicz S.: Sur le probléme de la division. Stud. Math. 8, 87-136 (1959) (or Rozpr. Mat. 22 (1961)) 
[M] Moser J.: Three integrable Hamiltonian systems connected with isospectral deformations. Adv. Math. 16, 1-23 (1975)

[O.P.] Olshanetsky M. A., Perelomov A. M.: Classical integrable finite-dimensional systems related to Lie algebras. Phys. Rep. 71, 5, 314-400 (1981)

[P] Perelomov A. M.: Equilibrium configuration and small oscillations of some dynamical systems. Ann. Inst. H. Poincaré (A) XXVIII, 4, 407-415 (1978)

Communicated by J. N. Mather

Received July 29, 1987; in revised form December 7, 1987 
